\title{
New features for the Journal
}

\author{
Richard D. Weisel, MD
}

Each month in this space we plan to provide the readers important information about the Journal, the AATS, and our specialty. In this edition of the notes, we will review the new instructions for authors and the expectation of the reviewers. In the near future, we will provide additional details about new initiatives at the Journal. Please watch this space in every issue.

As indicated in October's editorial, the Journal will publish high-impact papers. The new sections of the Journal will provide our readers the reasons why each paper was accepted and emphasize the importance of the results reported for our specialty. The initiatives include a "Perspective" and a "Central Picture" for all original manuscripts. In addition, we will publish an "Editorial Commentary" with nearly every paper. Finally, in this addition of the notes, we will outline the expectations of reviewers of the Journal.

Authors will be asked to provide a Perspective on their work for the readers. This section will provide a brief overview of the field and indicate the relevance and significance of their paper's results, detailing how the findings fit into their area of interest. The perspective should signify to the readers why they should pay attention to the manuscript. For clinical studies, the perspective section should identify the significance of the findings for the field and how the authors anticipate that their results may impact clinical practice today or in the future. For basic science studies, this section should indicate the potential clinical relevance of the findings and how the implications may impact clinical practice some day in the future. Perspectives should be no more than 100 words and will be highlighted in a special box in the manuscript.

A Central Picture should be included with all papers. The authors should designate one of their figures or provide a separate picture that summarizes an important component of their manuscript. The purpose of the illustration is to provide a snapshot of the paper with a single memorable visual image. The central picture should be accompanied by an abbreviated legend.

An Editorial Commentary will be published with most papers in the Journal. These commentaries,

From the Division of Cardiac Surgery, Toronto General Hospital, Research Institute and the University of Toronto, Toronto, Ontario, Canada.

Disclosures: Author has nothing to disclose with regard to commercial support.

Address for reprints: Richard D. Weisel, MD, Division of Cardiac Surgery, Toronto General Hospital, 200 Elizabeth St, 4N-476, Toronto, Ontario, Canada M5G 2C4

(E-mail: rweisel@uhnresearch.ca).

J Thorac Cardiovasc Surg 2014;148:2446

$0022-5223 / \$ 36.00$

Copyright (c) 2014 by The American Association for Thoracic Surgery

http://dx.doi.org/10.1016/j.jtcvs.2014.10.001

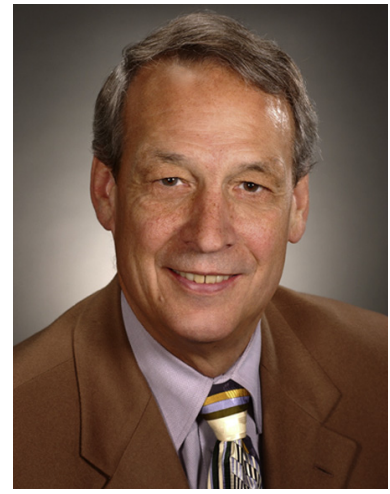

appearing right after a manuscript, will be written by an editor or reviewer to provide a broad perspective on the importance of the paper for the readers of the Journal. These contributions will specify why the paper is significant to the field and worthy of attention. In addition, the commentary will enumerate the strengths and weaknesses of the paper and suggest future studies required to move the field forward.

Reviewers are chosen by the Associate Editors for their expertise and their contributions to the field. Reviewer Expectations include an in-depth assessment of each paper and constructive recommendations to improve the quality and ensure that each accepted paper will make a significant impact on our specialty. The quality and utility of each review will be rated and included with the reviewer assessment. Timely response to invitations are also important expectations for reviewers. The goal is to provide a first response to the authors within 14 days following manuscript submission.

Editorial Board members are appointed from among the best reviewers. Most of these reviewers have provided highquality responses for at least 20 papers per year. In addition, they have positively responded to more than $90 \%$ of their invitations and provided excellent contributions in a timely manner for more than $80 \%$ of their reviews. Editorial Board members are expected to recommend papers that will generate at least 20 citations and 150 downloads in the first 18 months after publication.

The Journal will provide the readers with a deeper appreciation of the importance, relevance and significance of the published papers with Perspectives, Central Pictures and Editorial Commentaries. The success of the Journal is critically dependent on the excellence of the Associate Editors and reviewers. We will continue to provide transparent periodic performance evaluations to ensure that we are providing the best products for our readers. 